\title{
Polygraphic Studies of Normal Infants during the First Six Months of Life. I. Heart Rate and Variability as a Function of State
}

\author{
R. M. HARPER, ${ }^{(30)}$ TOKE HOPPENBROUWERS, M. B. STERMAN, D. J. MCGINTY, AND \\ J. HODGMAN \\ Sepulveda Veterans Administration Hospital, Departments of Anatomy, Psychiatry, and Psychology, UCLA and \\ Newborn Division of LAC-USC Medical Center, and Department of Pediatrics, USC School of Medicine, \\ Los Angeles, California, USA
}

\begin{abstract}
Extract
This study examined spontaneous heart rate $(\mathrm{HR})$ and variability as a function of age and sleep state in eight normal full term infants from birth to 6 months of age. Heart rates recorded during sleep were lower and less regular at 1 week (quiet sleep (QS) mean rate = 128, interquartile range $=6.4$ beats $/ \mathrm{min}$; rapid eye movement $(\mathrm{REM})=134.5,11.6)$ than at 1 month $(\mathrm{QS}=138.6,3.4 ; \mathrm{REM}$ 139.6, 4.2). Rate decreased sharply from 1 to 3 months $(Q S=118$; REM 123.8) and decreased only slightly thereafter (6-month QS = 113.5; REM 118.9). Variability decreased rapidly in REM from 2 to 4 months (from 11.4 to 9.1 ) and less quickly from 4 to 6 months (from 9.1 to 8.2), while QS variability decreased at 1 month (from 6.4 to 5.7$)$ and became stable from that point (6.0 at 6 months). Waking heart rate and variability were both relatively low at 1 week $(163,11.2 \mathrm{beats} / \mathrm{min})$ and increased from that age to 1 month $(167.4,14.3)$. Rate decreased rapidly in waking at 3 months (152 beats/min) and more slowly thereafter ( 152 beats/min at 4 months, 149 beats/min at 6 months), whereas variability remained elevated until after 3 months, becoming stable at a lower level during later infancy ( 3 months $=14.8,6$ months 11.7 ). Lowest values of rate and variability were found in $Q S$ and the highest values were found in waking at all ages, except at 1 week. Heart rates during REM closely approximated those in $\mathrm{QS}$, whereas variability values more closely resembled those of waking.
\end{abstract}

\section{Speculation}

It would appear that, in the normal infant, there are at least three relatively discrete stages in the ontogenetic sequence of cardiac rate and variability characteristics: a newborn period, early infancy (1-3 months), and later infancy. Since regulation of cardiac activity is greatly modified by sleep and waking behavior, the measurement of heart rate and variability must consider state as a factor in such regulation. Moreover, since states undergo both qualitative and quantitative changes during the first 6 months of age, the nature of cardiac regulation during this period may be a function of state maturation.

Normative heart rate data from the newborn period have been reported by several research groups $(2,11-18)$. However, data from older infants have largely been collected incidentally in the form of baseline levels during studies focused upon heart rate stimulus-response characteristics. Thus, Lipton et al. (10) provided individual HR levels before and after stimulation of infants at 2.5 and 5 months, from which a normative range and variability index could only be inferred. Lewis et al. (8) and Campos and Brackbill
(3) also presented cardiac data for infants between 2 and 8 weeks and 2 and 4 weeks with sample baseline rates. In addition, Lewis $e t$ al. (9) followed resting heart rate and variability from the prenatal period through the first year of life. The sequential development of cardiac regulation postnatally, except for these latter studies, is relatively unexplored.

Few studies, either of newborns or older infants, have systematically investigated the significant modulatory influence of sleep and waking states on cardiac parameters in infants. Prechtl et al. (11) reported that heart rates during QS and during REM or active sleep (AS) were similar in infants up to 8 days of age. The variability in heart rate, however, was twice as large during AS compared with QS. States also have a profound effect on cardiac response to tactile or auditory stimuli. Campos and Brackbill (3) have found that HR accelerations during AS were larger than those elicited in the quiet awake state, even after prestimulus effects were partialled out.

The modulation of heart rate and variability in relation to other periodic influences remains to be determined. Ashton and Connolly (2) showed, for example, that the feeding cycle affected heart rate during QS in neonates. The first QS period after feeding showed higher HR values than subsequent periods. This relationship was not observed during AS. Prechtl et al. (11) noticed an increase in HR during nutritive sucking, a decline afterwards, and a gradual increase toward a peak value within $30-50 \mathrm{~min}$, irrespective of subsequent state patterns.

In order to compile a comprehensive description of ECG parameters in developing infants, it is necessary to have long term physiologic monitoring so that an adequate number of sleep states are sampled and effects of situational adaptation and feeding are described. The present study, the first in a series, will deal with a description of heart rate and variability as a function of age and state in normal, full term infants from birth to 6 months of age.

\section{MATERIAL AND MONITORING PROCEDURE}

Eight neurologically normal infants of gestational ages between 39 and 41 weeks, and with 1-min Apgar scores of 8 or 9, participated in this study (Table 1). Five were males and three were females. Birth weights ranged from 3,040 to 4,270 g. Each infant was admitted at 5 PM to the sleep laboratory for 12-hr all-night monitoring sessions during the first week of life and at 1, 2, 3, 4, and 6 months of age. The parents were informed of the nature and objectives of the study and written permission was obtained before participation.

The infants were fed during preparation for monitoring and application of electrodes (see below). Newborn infants were swaddled and elbow restraints were applied to older infants before 
Table 1. Mean and SD of postnatal ages in eight normal full term infants at time of monitoring

\begin{tabular}{ccc}
\hline Monitoring interval & Mean age, days & SD \\
\hline 1 week & 4.8 & 2.0 \\
1 mo & 32.4 & 4.3 \\
2 mo & 62.4 & 3.8 \\
3 mo & 94.1 & 8.2 \\
4 mo & 125.2 & 10.5 \\
6 mo & 177.0 & 13.1 \\
\hline
\end{tabular}

initiation of recording. Monitoring was carried out in a darkened room which was separate from the recording equipment. Room temperature ranged from $23-25^{\circ}$. A demand feeding schedule was followed and, in several instances, the infant was breastfed by the mother. This did not interrupt the monitoring sequence. The infants were observed continuously during recording with the use of a low illumination camera and video monitor. Behaviors such as closing or opening of eyes, startles, crying, and vocalization were charted on the polygraphic paper.

\section{PHYSIOLOGIC RECORDING METHOD}

Both sleep and cardiopulmonary variables were monitored. The former included two EEG derivations together with chin EMG, eye movements, and gross somatic activity recordings. Two sets of Grass silver-silver chloride cup electrodes filled with Grass EC2 paste were applied bilaterally to the scalp at 2.5 and 6 $\mathrm{cm}$ lateral to vertex $(21)$. Cup electrodes were applied also to the skin overlying the mental protuberance and the belly of the digastric muscle. The head and chin were subsequently wrapped with an elastic gauze. An infrared transducer was used to monitor eye movements (4). Gross somatic activity was recorded through Mylar-coated conductive sensors positioned under the crib mattress. Thoracic or abdominal excursions were monitored with an impedance pneumograph (22). The respiratory rate was measured by means of a $\mathrm{pCO}_{2}$ monitor which sampled expired gas through a miniature cannula taped under the infant's nostrils (23). The ECG was recorded with two disposable electrodes placed symmetrically beneath the clavicles. A ground electrode was applied above the umbilicus. Additionally, a skin temperature probe was applied to the abdomen below the right costal margin.

Data were recorded on a 16-channel Grass model 78 polygraph (21) and simultaneously stored on a 14-channel analog tape recorder (24), together with an IRIG E time code (25). A detailed description of these methods will be published elsewhere (6).

\section{ANALYTIC METHODS}

Data on the analog tapes were processed with a PDP-12 computer (26) as follows. The $\mathrm{R}$ wave of the ECG signal was detected by an electronic peak searching discriminator which output a pulse on detection. The output pulse was sampled by the a/d converter of the PDP-12 at 512 times a second. Intervals between successive $R$ waves were calculated and stored on industry compatible magnetic tape, together with digitized values of the other physiologic measures. These remaining physiologic measures, which included EMG, motility, and respiratory activity, were taken because the description of these measures aided in the definition of state and helped in the recognition of those time periods which were contaminated by artifacts.

Heart rate values were calculated by measuring the median $R-R$ interval for each minute, and converting this value to rate. Heart rate variability was measured by determining the interquartile (IQ) range of $R-R$ intervals for each minute and converting these values to beats per min. Minute by minute values for median cardiac rate and variability over the entire $12 \mathrm{hr}$ ( 720 successive data values) were plotted on an incremental plotter (27). The median and interquartile range were chosen as appropriate measures since these two statistical procedures are relatively insensitive to occasional very deviant values caused by missed beats or interfering noise.

Each minute of the record was coded by trained personnel into quiet sleep (QS), active or REM sleep (REM), waking (AW), or transitional state ( $\mathrm{Tr}$ ), using state criteria and decision-making rules listed in Table 2 . The state scorers were trained to achieve a percentage agreement on state selection of $80 \%$. The criteria for state selection were similar to those described by Anders et al. (1) but, in addition, applied quantifiable values to each sleep parameter across an age range of 1 week to 6 months. The state codes were stored in a file on digital tape for correlation with heart rate and variability data. Subsequently, means of overall rate and variability measures for each state were calculated.

The ECG signal was occasionally contaminated with artifactual noise which, if included in the calculations, would give erroneous results. Artifacts manifested themselves principally as excessively long or short intervals. To deal with this problem, each R-R interval was plotted successively across the night, using a procedure described elsewhere (5). These plots were visually scanned, and those minutes in which the median would be significantly disturbed by many aberrant values were either removed or replaced with interpolated values based on preceding and following minutes. The use of median rather than mean values provided some degree of protection against aberrant data. In all cases the polygraphic records provided final reference for artifact evaluation. The percentage of minutes of data removed because of artifact contamination ranged from $0-8 \%$. Most contaminated data occurred in the waking state, whereas QS and REM state data were relatively unaffected. Except for those minutes of data removed because of contamination with artifacts, $R-R$ intervals over the entire $12-\mathrm{hr}$ record were used to derive results. Statistical assessment of the influence of age and state on heart rate was achieved by subjecting heart rate and variability values to analysis

Table 2. State scoring criteria and decision-making rules ${ }^{1}$

Code Criteria

REM Absence of sustained EMG tonus together with three of the following criteria:

1. At least 1 eye movement, independent of chin and gross body movements

2. Incidence of breathing rate increase or decrease larger than 25 breaths/min as measured by the respiratory tachometer.

3. Presence of twitches and brief head movements.

4. Absence of EEG spindles or tracé alternant.

QS All of the following criteria must be fulfilled:

1. Incidence of breathing rate increase and/or decrease equal to or less than 25 breaths/min as measured by respiratory tachometer. Ignore variability due to an apnea or isolated gross movement.

2. No more than one isolated eye movement. Eyes closed.

3. Sustained EMG tonus and/or EEG spindles or tracé alternant.

AW Three of the following criteria must be fulfilled:

1. Sustained EMG tonus with activity bursts.

2. Eyes open.

3. Irregular respiration; variability larger than 45 breath $/ \mathrm{min}$ as measured by respiratory tachometer.

4. Vocalization.

5. Sustained gross movements.

Tr Minutes in which the criteria for REM, AS and QS are not fulfilled, or minutes in which these criteria are fulfilled for less than 30 consecutive sec.

'REM: rapid eye movement; QS: quiet sleep; AW: active sleep; $\mathrm{Tr}$ : transitional. 
of variance procedures. Developmental trends were analyzed by the method of orthogonal polynomials.

\section{RESULTS}

\section{HEART RATE}

Heart rate data at each age exhibited periodic modulation across the night. Examples of this are presented in Figure 1, which shows successive 12 -hr plots of minute by minute median heart rate in one infant across the six age periods sampled. A rhythmic modulation of cardiac activity with a period approximating $60 \mathrm{~min}$ is apparent in each plot. This modulation was directly correlated with alternation of sleep-waking patterns during the night (Fig. 2). Heart rate was lowest during QS and highest during $\mathrm{AW}$, with intermediate values during REM.

\section{HRMD}

1 WK

225

$1 \mathrm{MO}$

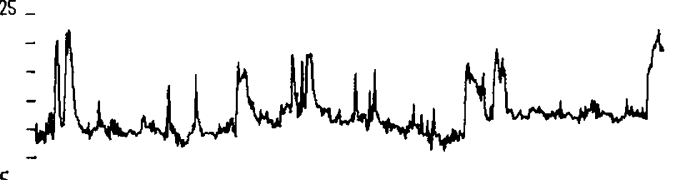

75

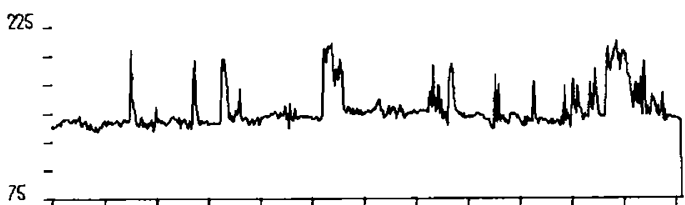

225

$2 M O$

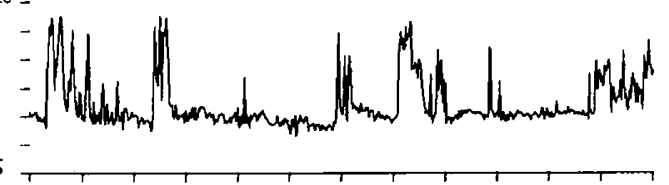

$3 M O$

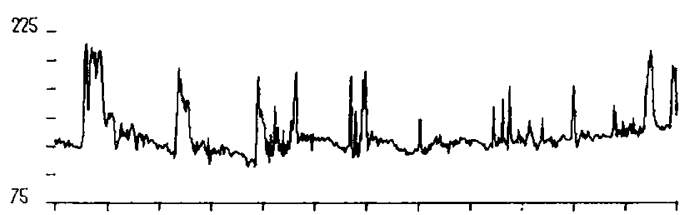

225

4 MO

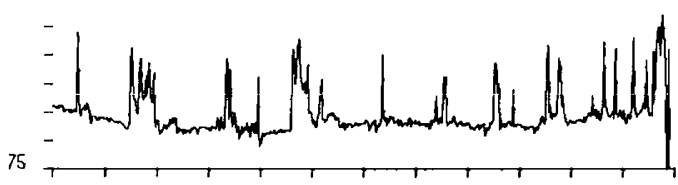

$6 M O$

225

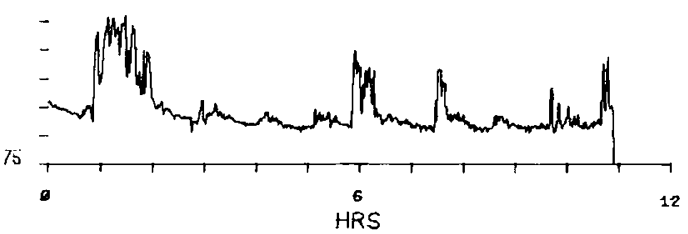

Fig. 1. Minute by minute median heart rate values for 12 -hr recording periods at six ages. The data are derived from one normal individual and illustrate some of the periodic modulations of heart rate that occur in long term recordings.
The data presented in Figure 1 also serve to illustrate developmental trends. Summary data for each infant at each age and state are listed in Table 3. Mean values for all infants are shown collectively in Figure 3. A mixed model analysis of variance (ANOVA), coupled with a trend analysis, was used to examine these data. Heart rate medians showed a significant overall linear decline with age in all states $(P<0.001)$. This linear decline in part reflected the tendency for values to differ before and after 3 months of age. However, this linear trend was not sufficient to describe the data. A significant cubic component $(P<0.01)$, related to an increase in rate up to 1 month, contrasted with a general decrease and leveling off afterwards

\section{RATE VS. PROFILE}

Sleep-waking states exerted an important influence upon heart rate. Although developmental changes were apparent, the mixed model ANOVA showed that the profile of differences between states was retained with age. A Duncan's Multiple Range Test showed that group mean values were significantly different among the three primary state patterns, AW, QS, and REM $(P<0.05)$, with heart rates consistently highest during waking. Transition state values were significantly different from those during AW and QS $(P<0.01)$ but not during REM.

To illustrate rate trends in individuals and the variability among individuals in these measures, the values for each individual are plotted by age and by state in Figure 4 . The greatest variability between individuals in all states, except $\mathrm{AW}$, was observed in the first week of life, whereas the lowest variability, again with the exception of waking, was registered 3 weeks later, at 1 month of age. From that point on, group variability remained more or less constant and comparable among the state patterns. The waking state was unusual in that a large variability among infants appeared at 1 month and then again after 4 months of age.

\section{VARIABILITY}

Heart rate variability within subjects was measured by calculating the interquartile range of $\mathrm{R}-\mathrm{R}$ intervals for each minute and converting these values to beats per min. Variability also exhibited periodic modulation during the night. This is illustrated in Figure 5 , which shows 12 -hr plots of successive minute by minute heart rate variability in one infant across the six ages sampled. In this figure, increased variability is represented by upward deflections in the plot. During each age period one of the most striking features of the data was their obvious periodicity, with a recurrent cycle

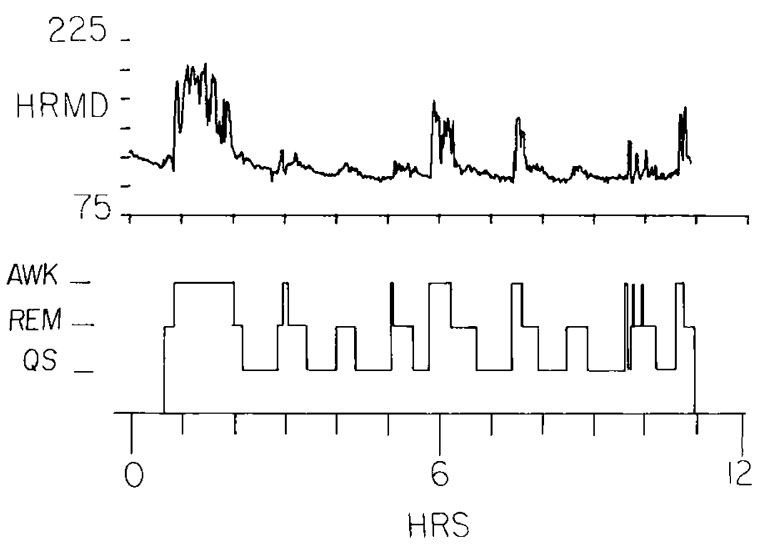

Fig. 2. Median heart rate (top) and the accompanying sequence of sleep states (bottom) in a normal 6-month-old individual over an 11-hour recording period. There are regularly recurring periods of decreased rate which are associated with epochs of quiet sleep $(Q S)$, as well as periods of increased rate which are associated with waking. $R E M$ : rapid eye movement; $A W K$ : awake. 
Table 3. Median individual heart rate $(H R)$ during sleep and waking states ${ }^{1}$

\begin{tabular}{|c|c|c|c|c|c|c|}
\hline \multirow{2}{*}{$\begin{array}{c}\text { State } \\
\text { and } \\
\text { subjects }\end{array}$} & \multicolumn{6}{|c|}{ HR (beats/min) at various ages } \\
\hline & $1 \mathrm{wk}$ & l mo & $2 \mathrm{mo}$ & $3 \mathrm{mo}$ & $4 \mathrm{mo}$ & $6 \mathrm{mo}$ \\
\hline \multicolumn{7}{|l|}{ QS } \\
\hline 1 & 114.6 & 141.0 & 132.0 & 124.4 & 121.1 & 102.6 \\
\hline 2 & 126.0 & 145.0 & 124.9 & 125.9 & 112.7 & 109.4 \\
\hline 3 & 131.8 & 136.4 & 129.0 & 116.1 & 110.5 & 112.8 \\
\hline 4 & 139.8 & 133.2 & 125.1 & 115.1 & 116.5 & 109.5 \\
\hline 5 & 136.3 & 137.4 & 138.5 & 122.7 & 122.0 & 114.7 \\
\hline 6 & 114.5 & 137.3 & 136.4 & 114.5 & 134.5 & 121.1 \\
\hline 7 & 121.5 & 134.7 & 123.5 & 120.4 & 124.3 & 118.5 \\
\hline 8 & 139.8 & 137.9 & 128.2 & 111.3 & 105.9 & 118.9 \\
\hline Mean & 128.0 & 138.6 & 129.7 & 118.8 & 118.4 & 113.5 \\
\hline SD & 10.5 & 3.4 & 5.5 & 5.3 & 9.0 & 6.2 \\
\hline \multicolumn{7}{|l|}{ REM } \\
\hline 1 & 117.8 & 144.0 & 139.5 & 127.8 & 125.1 & 107.7 \\
\hline 2 & 131.3 & 145.7 & 126.1 & 132.8 & 117.9 & 115.7 \\
\hline 3 & 133.4 & 139.5 & 132.0 & 120.2 & 116.9 & 116.0 \\
\hline 4 & 143.1 & 140.9 & 127.2 & 119.4 & 119.8 & 118.3 \\
\hline 5 & 143.3 & 138.7 & 137.3 & 126.6 & 126.1 & 120.7 \\
\hline 6 & 131.2 & 136.5 & 138.9 & 119.0 & 138.7 & 128.5 \\
\hline 7 & 128.4 & 132.2 & 126.3 & 122.0 & 125.9 & 121.7 \\
\hline 8 & 147.7 & 139.5 & 132.3 & 116.6 & 111.0 & 122.4 \\
\hline Mean & 143.5 & 139.6 & 132.4 & 123.1 & 122.7 & 118.9 \\
\hline SD & 9.7 & 4.2 & 5.6 & 5.5 & 8.3 & 6.2 \\
\hline \multicolumn{7}{|l|}{ AW } \\
\hline$l$ & 159.8 & 184.2 & 173.5 & 152.6 & 151.7 & 134.8 \\
\hline 2 & 164.2 & 168.2 & 179.8 & 161.3 & 153.2 & 160.3 \\
\hline 3 & 151.3 & 152.8 & 162.6 & 147.3 & 143.4 & 122.6 \\
\hline 4 & 174.4 & 179.5 & 172.3 & 146.4 & 138.7 & 146.0 \\
\hline 5 & 156.3 & 159.1 & 166.4 & 149.7 & 145.6 & 162.8 \\
\hline 6 & 149.7 & 151.6 & 162.7 & 149.8 & 159.8 & 158.7 \\
\hline 7 & 157.5 & 173.1 & 150.7 & 146.8 & 161.9 & 140.0 \\
\hline 8 & 191.0 & 170.6 & 169.1 & 162.4 & 162.7 & 167.5 \\
\hline Mean & 163.0 & 167.4 & 167.1 & 152.0 & 152.1 & 149.0 \\
\hline $\mathrm{SD}$ & 13.7 & 12.0 & 8.8 & 6.4 & 8.0 & 15.8 \\
\hline \multicolumn{7}{|l|}{$\operatorname{Tr}$} \\
\hline 1 & 137.9 & 156.6 & 142.3 & 129.0 & 127.0 & 106.1 \\
\hline 2 & 131.6 & 149.4 & 142.2 & 131.3 & 116.4 & 116.5 \\
\hline 3 & 137.5 & 141.7 & 142.0 & 121.0 & 117.6 & 117.8 \\
\hline 4 & 137.9 & 144.6 & 149.0 & 117.0 & 124.6 & 122.6 \\
\hline 5 & 135.4 & 138.0 & 136.1 & 125.4 & 125.3 & 118.0 \\
\hline 6 & 127.2 & 142.7 & 139.0 & 116.7 & 138.3 & 125.5 \\
\hline 7 & 123.7 & 150.6 & 127.1 & 126.2 & 125.2 & 121.0 \\
\hline 8 & 161.1 & 144.3 & 135.2 & 113.9 & 109.9 & 126.2 \\
\hline Mean & 136.5 & 146.0 & 139.1 & 122.6 & 123.0 & 119.2 \\
\hline SD & 11.3 & 5.9 & 6.5 & 6.4 & 8.5 & 6.4 \\
\hline
\end{tabular}

${ }^{1} \mathrm{QS}$ : quiet sleep; REM: rapid eye movement; AW: awake; $\mathrm{Tr}$ : transitional.

approximating $60 \mathrm{~min}$. This cycle was related directly to the periodic sequence of sleep and waking states during the all-night recordings (Fig. 6). In waking states, variability was closely associated with behavior, such that crying, fussing, and gross somatic activity resulted in sustained increases, whereas feeding or quiescence produced relatively stable cardiac activity. During sleep, variability was modulated in relation to the alternating cycle of REM-QS periods, with low and stable values during QS and consistently high variability during REM.

Median variability values for each infant during each state and age are listed in Table 4 . Group means for these values are plotted in Figure 7 . This figure demonstrates that heart rate variability
HEART RATE

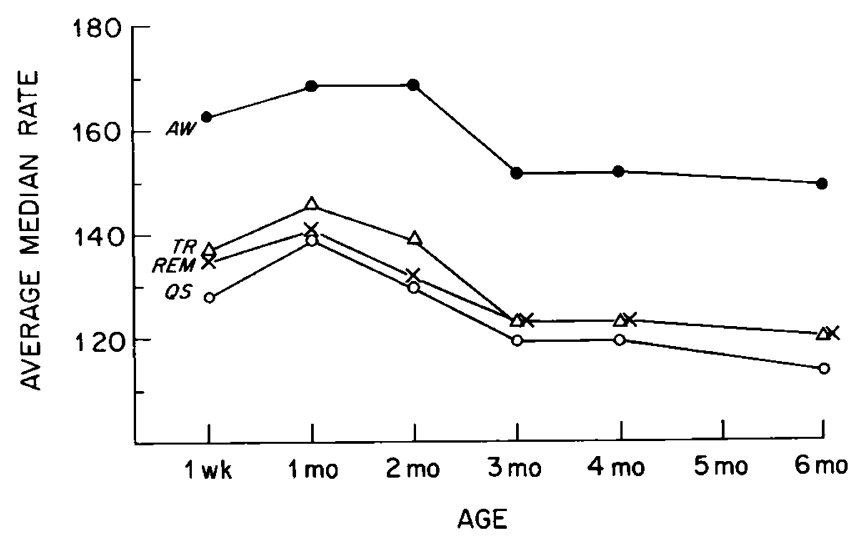

Fig. 3. Averaged median rates for all individuals over six age periods during four different states.
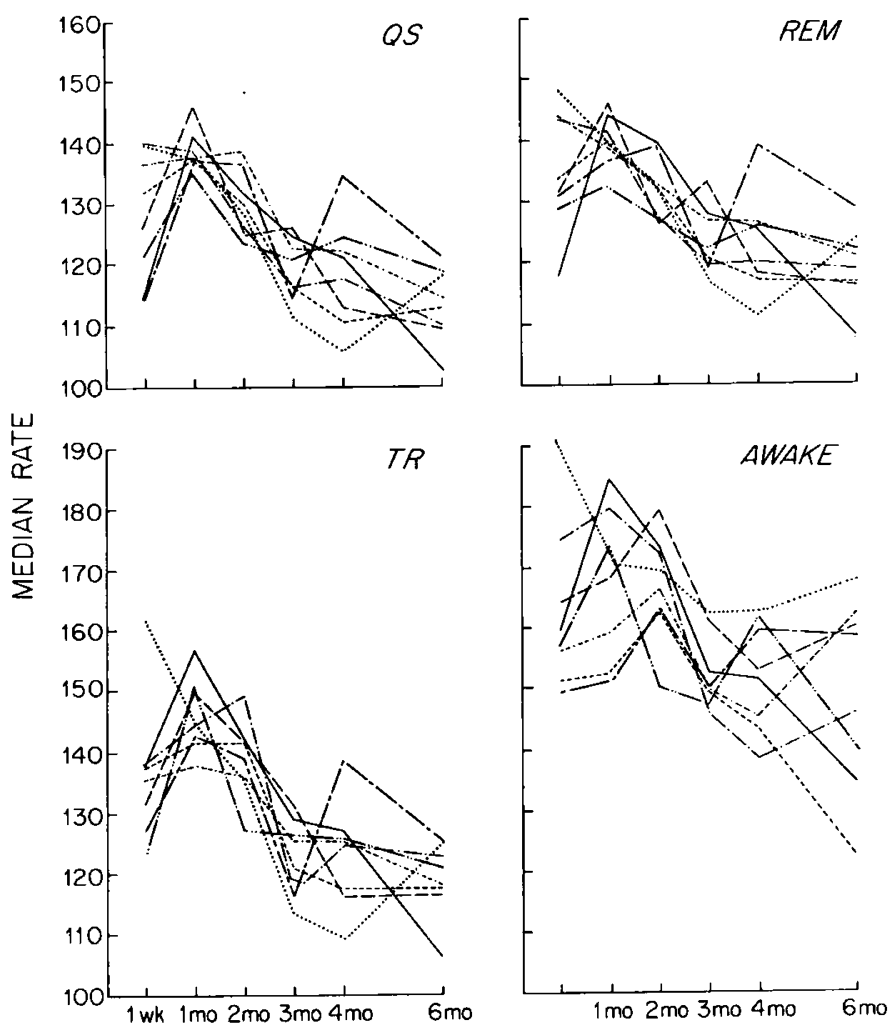

Fig. 4. Averaged median rates for each individual at each age for each state. $Q S$ : quiet sleep; $R E M$ : rapid eye movement; $\operatorname{Tr}$ : transitional state.

was highest in AW at all ages except 1 week and lowest in QS at all ages. Variability during REM and Tr states was intermediate, but more closely resembled the waking state levels. Statistical analysis of these curves by a mixed model ANOVA indicated that the overall differences among both ages $(P<0.05)$ and states $(P<$ $0.01)$ were significant. In addition, a Duncan's Multiple Range Test indicated significant differences between each state $(P<$ 0.01 ), with the exception that $\operatorname{Tr}$ and REM were indistinguishable. There was a significant interaction of state and age factors $(P<$ 0.01 ), however. One contributing source of this interaction was the deviant rank order among the states in the l-week means. This suggests that the rank order of states demonstrated by the Duncan test may not be true at 1 week.

Figure 7 illustrates the developmental progression of heart rate variability. Since a major part of the age-state interaction was suspected to derive from the stability of QS variability across age, an analysis of variance omitting the QS data was calculated. The 
VAR

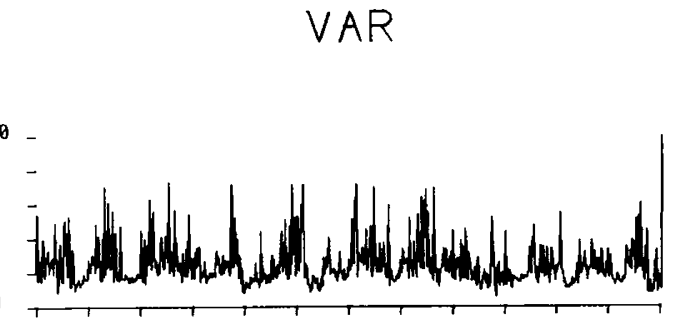

1 WK$$
0
$$

$1 \mathrm{MO}$

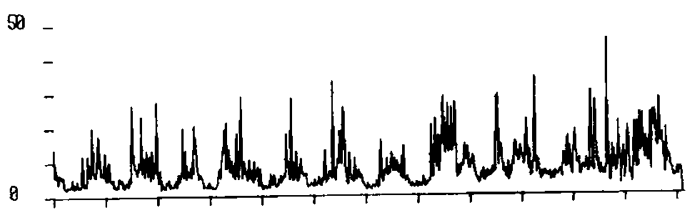

$2 M O$

50

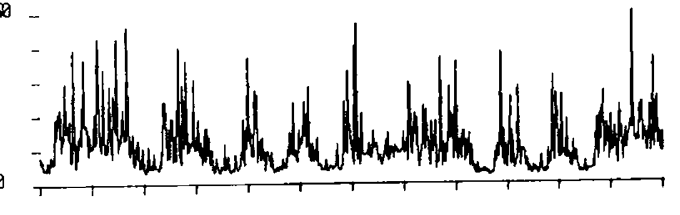

$3 M O$

So -

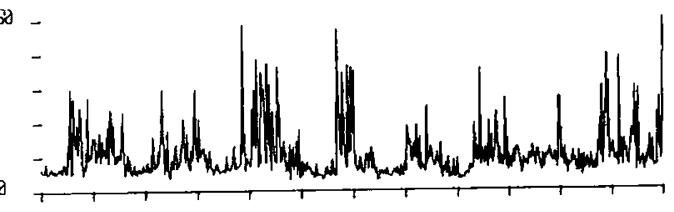

$4 M O$

$50-$

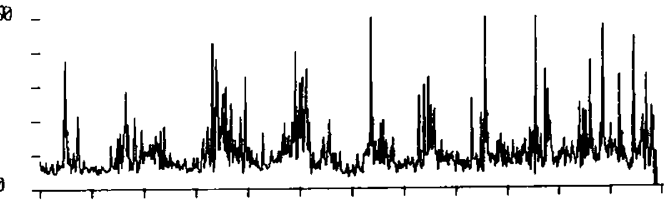

so -

6 MO

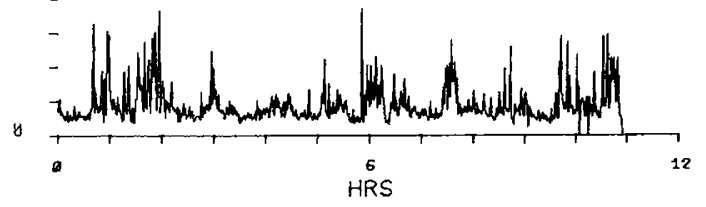

Fig. 5. Interquartile range values for successive minutes over 12-hr periods at six ages from a single individual.

interaction of age and state no longer attained significance once these data were excluded. A trend analysis conducted on the development of the remaining three states indicated a significant quadratic component with its peak at 2 months $(P<0.01)$. This finding reflected the obvious departure from linearity in $\mathrm{AW}, \mathrm{Tr}$, and REM states during this period. Waking variability was clearly increased, and a similar trend was apparent for $\mathrm{Tr}$ and REM values. Variability during QS was greatest at 1 week, but appeared to be relatively stable from that point forward.

The mean of the median variability values for each infant at each age and for each state is also shown in Table 4. In QS and REM, differences appeared to decline with age, with the infants being very similar in variability at $1-2$ months. Between-subject variability in the AW state was minimal at 1 week of age, increased markedly by 2 and 3 months, and declined slightly thereafter.

\section{DISCUSSION}

It is clear that any attempt to evaluate cardiac rate and variability measures in the infant must consider the functional

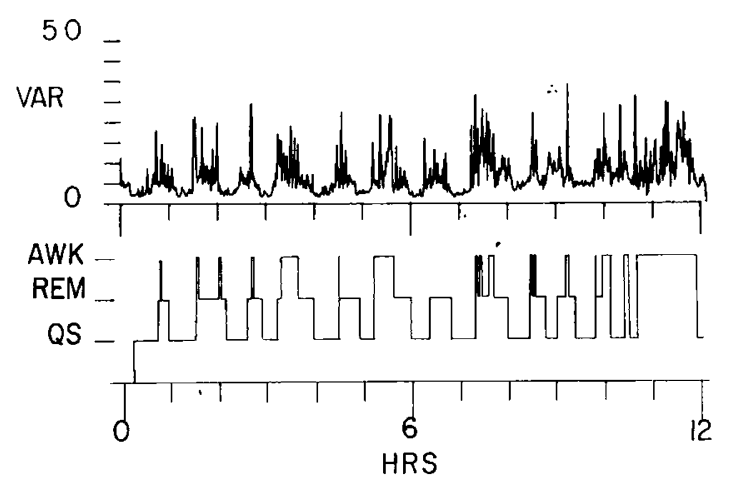

Fig. 6. Interquartile range (top) and the accompanying sequence of sleep states (bottom) in a normal 6-month-old individual over a 12-hr recording period. There are regularly recurring periods of decreased variability which are associated with quiet sleep $(Q S)$, as well as periods of increased variability associated with epochs of waking $(A W K)$ and rapid eye movement $(R E M)$.

state or states from which the data were obtained. Although significant developmental changes occurred, the characteristic relationship among values in the various state patterns was retained, indicating that physiologic organization associated with state remains a primary influence throughout development. Heart rates were consistently highest and most variable during waking. This was because of the high incidence of movement and arousal associated with the fussing, feeding, crying, etc., which was characteristic of this state. Conversely, values during QS were lowest at all ages. The decrease in variability during this state was particularly striking.

Heart rates during REM closely approximated those in QS, whereas variability was clearly much greater in REM. Thus, from the standpoint of rate REM was similar to QS, but resembled waking in terms of variability. The neural factors mediating these two parameters of cardiac activity would appear to be at least partially independent.

It would appear that there are at least three relatively discrete stages in the ontogenetic sequence of cardiac rate and variability characteristics. Overall patterns indicated a separation between the newborn period ( 1 week), early infancy ( $1-3$ months), and later infancy (over 3 months). Heart rates during sleep were lower and less regular at 1 week when compared with 1 month. Rate tended to decrease sharply from 1 to 3 months, and decreased only slightly thereafter. Variability decreased rapidly in REM from 2 to 4 months and less quickly from 4 to 6 months, whereas QS variability decreased at 1 month and was essentially stable from that point onward. Waking heart rate and variability were both lower at 1 week than at 1 and 2 months. Rate decreased precipitously in waking at 3 months and more slowly thereafter, whereas variability remained elevated until after 3 months, then stabilized at a lower level during later infancy.

Differences among infants in both rate and variability were greatest at 1 week and least at 1 month for QS and REM. However, differences among infants in waking declined for rate but not for variability over the first month. Kero (7) and Valimaki et al. (16) have noted that heart rate and variability increase during the first 5 days of life. Thus, part of the between-individual variation at 1 week may be related to our sampling over that entire period. Differences in rate between individuals during sleep increased again at 2 months and then showed a slight decline gradually with age. A similar pattern was seen in the within-subjects variability data. During wakefulness differences among infants declined again after 1-2 months, with rates becoming more diverse thereafter and variability values showing increasing stability.

Comparisons of these results with other heart rate and variability studies of developing infants is difficult, since most such studies were carried out as stimulus response experiments $(3,8,10,19)$, or 
Table 4. Individual heart variability (interquartile range) during sleep and waking states ${ }^{1}$

\begin{tabular}{|c|c|c|c|c|c|c|}
\hline \multirow{2}{*}{$\begin{array}{c}\text { State } \\
\text { and } \\
\text { subjects }\end{array}$} & \multicolumn{6}{|c|}{$\mathrm{HR}$ (beats $/ \mathrm{min}$ ) at various ages } \\
\hline & $1 \mathrm{wk}$ & $1 \mathrm{mo}$ & $2 \mathrm{mo}$ & $3 \mathrm{mo}$ & $4 \mathrm{mo}$ & $6 \mathrm{mo}$ \\
\hline \multicolumn{7}{|l|}{ QS } \\
\hline 1 & 3.1 & 3.8 & 3.0 & 4.9 & 2.2 & 5.5 \\
\hline 2 & 8.9 & 4.7 & 6.3 & 6.8 & 7.0 & 6.3 \\
\hline 3 & 4.9 & 7.1 & 3.3 & 4.0 & 5.6 & 4.8 \\
\hline 4 & 6.1 & 4.7 & 5.5 & 7.1 & 6.5 & 6.1 \\
\hline 5 & 5.6 & 5.3 & 2.5 & 3.1 & 3.5 & 5.8 \\
\hline 6 & 5.3 & 6.6 & 6.5 & 7.9 & 7.3 & 8.0 \\
\hline 7 & 7.3 & 5.8 & 6.8 & 6.3 & 6.5 & 5.4 \\
\hline 8 & 10.0 & 7.3 & 9.8 & 8.1 & 6.1 & 5.7 \\
\hline Mean & 6.4 & 5.7 & 5.5 & 6.0 & 5.6 & 6.0 \\
\hline SD & 2.3 & 1.3 & 2.5 & 1.8 & 1.8 & 0.98 \\
\hline \multicolumn{7}{|l|}{ REM } \\
\hline 1 & 8.7 & 9.3 & 7.3 & 9.1 & 4.7 & 8.0 \\
\hline 2 & 13.7 & 8.3 & 12.3 & 11.3 & 10.9 & 8.5 \\
\hline 3 & 8.8 & 8.9 & 10.5 & 8.3 & 9.0 & 7.8 \\
\hline 4 & 12.7 & 11.4 & 12.5 & 11.4 & 8.8 & 9.2 \\
\hline 5 & 10.6 & 9.6 & 9.3 & 8.6 & 6.0 & 7.1 \\
\hline 6 & 9.8 & 11.5 & 12.0 & 12.0 & 10.4 & 9.4 \\
\hline 7 & 12.9 & 13.8 & 11.9 & 13.3 & 12.8 & 9.6 \\
\hline 8 & 16.0 & 12.9 & 15.7 & 12.6 & 10.4 & 8.2 \\
\hline Mean & 11.6 & 10.7 & 11.4 & 10.8 & 9.1 & 8.2 \\
\hline SD & 2.6 & 2.0 & 2.5 & 1.9 & 2.7 & 1.4 \\
\hline \multicolumn{7}{|l|}{$\mathrm{AW}$} \\
\hline$l$ & 11.9 & 9.5 & 9.3 & 11.9 & 8.9 & 13.0 \\
\hline 2 & 11.8 & 13.3 & 15.2 & 15.6 & 17.2 & 12.9 \\
\hline 3 & 7.8 & 10.7 & 14.1 & 12.6 & 11.4 & 11.8 \\
\hline 4 & 11.1 & 12.8 & 14.6 & 14.7 & 12.9 & 15.5 \\
\hline 5 & 10.6 & 7.7 & 8.4 & 9.0 & 5.8 & 8.2 \\
\hline 6 & 11.8 & 13.4 & 17.2 & 13.7 & 13.2 & 8.9 \\
\hline 7 & 11.4 & 16.6 & 21.1 & 26.4 & 14.2 & 11.3 \\
\hline 8 & 13.1 & 14.0 & 14.6 & 14.5 & 12.0 & 11.8 \\
\hline Mean & 11.2 & 12.2 & 14.3 & 14.8 & 12.0 & 11.7 \\
\hline SD & 1.6 & 2.8 & 4.1 & 5.1 & 3.4 & 2.3 \\
\hline \multicolumn{7}{|l|}{$\operatorname{Tr}$} \\
\hline 1 & 11.1 & 9.0 & 7.6 & 8.0 & 5.0 & 8.4 \\
\hline 2 & 11.8 & 8.9 & 15.7 & 12.4 & 10.7 & 8.9 \\
\hline 3 & 8.0 & 11.5 & 12.7 & 13.1 & 10.1 & 11.1 \\
\hline 4 & 9.9 & 11.0 & 13.0 & 12.6 & 11.9 & 13.8 \\
\hline 5 & 9.8 & 9.4 & 10.0 & 5.7 & 9.2 & 7.5 \\
\hline 6 & 8.5 & 10.8 & 14.0 & 12.4 & 12.7 & 12.1 \\
\hline 7 & 13.0 & 16.4 & 11.7 & 20.9 & 10.0 & 8.9 \\
\hline 8 & 15.4 & 14.3 & 18.1 & 11.5 & 9.1 & 10.5 \\
\hline Mean & 10.9 & 11.4 & 12.9 & 12.1 & 9.8 & 10.2 \\
\hline SD & 2.4 & 2.7 & 3.3 & 4.4 & 2.3 & 2.1 \\
\hline
\end{tabular}

${ }^{1} \mathrm{HR}$ : heart rate; QS: quiet sleep; REM: rapid eye movement; AW: awake; Tr: transitional.

did not consider state as a factor in controlling rate and variability $(7,9,13,15-18)$.

Lewis et al. (9) found a linear decrease in "resting" heart rate over the first year of life, and also showed a slight increase in rate and variability at 1 month of age. Their values for rate fall between 152 beats $/ \mathrm{min}$ at 2 weeks to 147 beats/min at 6 months, values slightly lower than those for rate during waking in our study. These lower rates probably result from the inclusion of both waking and sleep values in the "resting" measures.

Watanabe et al. (20) present data for heart rate and variability
VARIABILITY

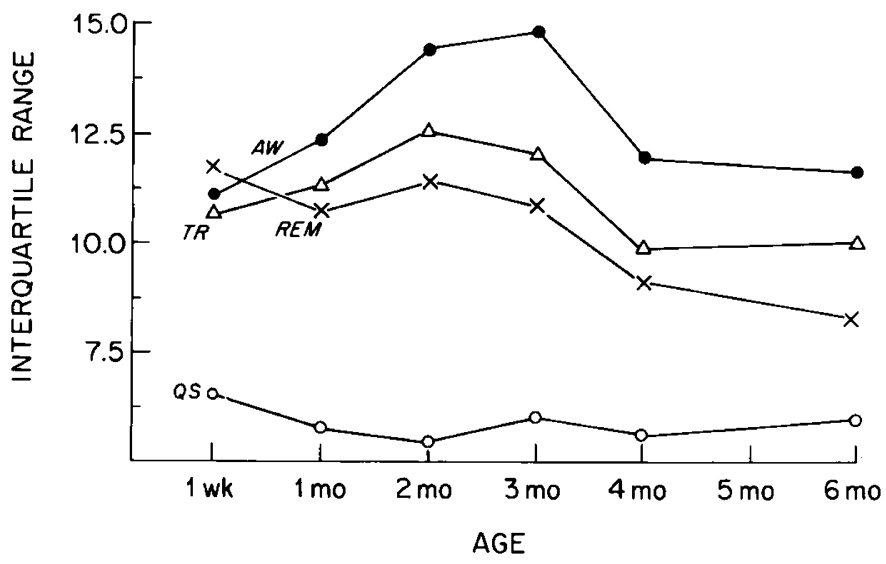

Fig. 7. Averaged interquartile ranges of heart rate variability for eight normal infants from birth to 6 months of age. $A W$ : awake; $T R$ : transitional; $R E M$ : rapid eye movement.

during sleep over the first 30 weeks of life. Unfortunately, an evoked response experiment was simultaneously being performed while the baseline data were being collected, which might grossly affect spontaneous rate and variability. Their data do indicate, however, a decrease in $\mathrm{R}-\mathrm{R}$ interval (i.e., increase in rate) from birth to the first month, followed by a gradual increase in $\mathrm{R}-\mathrm{R}$ interval (decrease in rate), which is similar to the results described here.

Neuronal maturation in the first month of life appears to favor increased cardiac regulation during sleep states together with increased responsiveness during waking. From 1-3 or 4 months of age a second stage in development emerged, characterized by an increase and then a decrease in rate and variability in all states except QS, which showed minimal and unchanging variability after 1 month. Finally, a third stage appeared after 3 months which was indicative both of mature regulatory control and the gradual expression of individual characteristics.

\section{SUMMARY}

Heart rate and variability during sleep and waking periods were examined in infants from 1 week to 6 months of age. Heart rates and variability were lowest in quiet sleep, whereas highest values were found in waking. Heart rates during both sleep and waking were lower and less regular at 1 week than 1 month and then decreased until 6 months. Variability in both waking and sleeping states was lower at 1 week than 1 month; after 1 or 2 months of age, depending on the state, variability again decreased, at first rapidly and then more slowly until 6 months. Rate during REM closely approximated rates in QS, whereas variability values more closely resembled those of waking.

\section{REFERENCES AND NOTES}

1. Anders, T., Emde, R., and Parmelee, A.: A Manual of Standardized Terminology, Techniques and Criteria for Scoring of States of Sleep and Wakefulness in Newborn Infants (UCLA Brain Information Service/BRI Publications Office. Los Angeles, 1971).

2. Ashton, R., and Connolly, K.: The relation of respiration rate and heart rate to sleep states in the human newborn. Develop. Med. Child Neurol., 13: 180 (1971).

3. Campos, J. J., and Brackbill, Y.: Infant state: Relationship to heart rate, behavioral response and response decrement. Develop. Psychobiol., 6(1): 9 (1973).

4. Harper, R. M., Hoppenbrouwers, T., and Ross, S. A.: A new technique for long-term recording of eye movements in infants. Electroenceph. Clin. Neurophysiol., 40: 109 (1976).

5. Harper, R. M., Mason, J., Pacheco, R., and Perga, A.: A computer system for the analysis of physiological data during sleep. (Submitted for publication: manuscript available on request.) 
6. Hofmann, E., Havens, B., Hoppenbrouwers, T., Geidel, S., and Hodgman, J.: Long-term continuous monitoring of multiple physiological parameters in newborn and young infants: A procedural manual (Submitted for publication; manuscript available on request.)

7. Kero, $P$. .: Heart rate variation in infants with the respiratory distress syndrome. Acta Pediat. Scand., Suppl. 250 (1974)

8. Lewis, M., Bartels, B., and Goldberg, S.: State as a determinant of infants' heart rate response to stimulation. Science, 155: 486 (1967)

9. Lewis, M., Wilson, C. D., Ban, P., and Baumel, M. H.: An exploratory study of resting cardiac rate and variability from the last trimester of prenatal life through the first year of postnatal life. Child Develop., 41: 799 (1970).

10. Lipton, E., Steinschneider, A., and Richmond, J.: Autonomic function in the neonate. VII. Maturational changes in cardiac control. Child Develop., 37(1):1 (1966).

11. Prechtl, H. F. R., Weinmann, H., and Akiyama, Y.: Organization of physiological parameters in normal and neurologically abnormal infants. Neuropediatrie, 1: 101 (1969).

12. Roffwarg, H. P., Muzio, J. N., and Dement, W. C.: Ontogenetic development of the human sleep-dream cycle. Science, 152: 604 (1966).

13. Tarlo, P. A., Valimaki, I., and Rautaharju, P. M.: Quantitative computer analysis of cardiac and respiratory activity in newborn infants. J. Appl. Physiol., 3/(1): 70 (197I).

14. Urbach, J. R.. Phuvichit, B., Zweizig, H., Millkan, E., Carrington, E. R., Loveland, M., Williams, J. M., Duncan, A. M., Farrell, S. L., Simons, P. O., and Spurgeon, I. L.: Instantaneous heart-rate patterns in newborn infants. Amer. J. Obstet. Gynecol., 93: 965 (1965).

15. Valimaki, I.: Heart-rate variation in full-term newborn infants. Biol. Neonate, 18: 129 (1971).

16. Valimaki, I., Rautaharju, P. M., Roy, S. B., and Scott, K. E.: Heart rate patterns in healthy term and premature infants and in respiratory distress syndrome. Eur. J. Cardiol., I(4): 411 (1974).

17. Valimaki, I., and Tarlo, P. A.: Heart rate patterns and apnea in newborn infants. Amer. J. Obstet. Gynecol., 110(3): 343 (1971).

18. Vallbona, C., Desmond, M. M., Rudolf, A. J., Papp, L. F., Franklin, R. R., and Rush, J. B.: Cardiodynamic studies in the newborn. II. Regulation of the heart rate. Biol. Neonate, 5: 159 (1963).

19. Vranekovic, G., Hock, E., Isaac, P., and Cordero, L.: Heart rate variability and cardiac response to an auditory stimulus. Biol. Neonate, 24: 66 (1974).

20. Watanabe, K., Iwase, K., and Hara, K.: Heart rate variability during sleep and wakefulness in low birthweight infants. Biol. Neonate, 22: 87 (1973).

21. Grass Instruments Co., Quincy, Mass.

22. Gould Inc., Instrument Systems Division, Los Angeles, Calif.

23. Beckman Instruments, Palo Alto, Calif.

24. Honeywell, Test Instruments Division, Denver, Colo.

25. Systron Donner Corp., Concord, Calif.

26. Digital Equipment Corp., Maynard, Mass.

27. Houston Instruments, Bellaire, Texas.

28. We would like to thank Dr. E. Gocka for his statistical help in these studies. D. Bannett, S. Geidel, B. Havens, D. Hockin, D. Kelly, and J. R. Mason also made valuable contributions to this study. Computational support was received from the Biomedical Engineering and Computing Center at the Sepulveda Veterans Administration Hospital.

29. This research was supported by the Veterans Administration and by the National Institute of Child Health and Human Development Contracts nos. N01HD-2-2777 and HD4-2810.

30. Requests for reprints should be addressed to: R. M. Harper, Ph.D., Department of Anatomy, University of California, Los Angeles, Calif. 90024 (USA).

31. Accepted for publication May 25, 1976.

\title{
Effect of Undernutrition and Amino Acid Deficiency on the Timing of Puberty in Rats
}

\author{
A. R. GLASS, R. HARRISON, AND R. S. SWERDLOFF 121 \\ Harbor General Hospital Campus, UCLA School of Medicine, Torrance, California, USA
}

Extract

Sexual maturation was examined in underfed or amino acid-deficient rats. We have demonstrated a highly 'significant negative linear relationship $(r=-0.80, P<0.001)$ between the age at puberty and the growth rate in rats under conditions of food restriction. The weight at puberty in animals growing at different rates because of undernutrition was not constant but behaved as a quadratic function of growth rate, as predicted from the assumption that growth rate was an independent variable. Growth rate is therefore more important than arrival at a particular fixed weight in determining the timing of puberty. Feeding of low valine diets resulted in delayed sexual maturation. Both the weight at vaginal opening $(182 \pm 5.9 \mathrm{~g})$ and the weight at first estrus $(187 \pm 6.1 \mathrm{~g})$ were significantly greater in the valine-deficient group when compared with growthmatched control $(139 \pm 10.7 \mathrm{~g}$ and $161 \pm 9.3 \mathrm{~g}$, respectively, $P<$ $0.05)$. The valine-deficient group also had significantly later vaginal opening $(98.8 \pm 4.7$ days) than growth-matched controls $(76.6 \pm$ 6.6 days, $P<0.02$ ). Valine deficiency seemed to have a specific effect on the hypothalamic-pituitary-gonadal axis since puberty in valine-deficient animals was delayed more than could be accounted for by impairment of growth.
Speculation

In contrast with previous suggestions in animals and man, we have shown that in rats the weight at puberty is not constant under conditions of underfeeding or valine deficiency. Growth rate seems to be more important than arrival at a particular fixed weight in determining the onset of puberty, possibly through a linkage between growth rate and hormone production rates. Valine deficiency may cause delayed puberty by altering central nervous system neurotransmitters.

The relationship between somatic growth and sexual maturation has been a topic of interest to clinicians and researchers for many years. Further excitement was generated in 1970 by the proposal of Frisch and Revelle that menarche in girls tends to occur at a critical constant body weight and that this phenomenon is important in understanding the hormonal basis of puberty $(3,4)$. This proposal was inspired in part by evidence suggesting that puberty in animals tended to occur at a constant body size. Subsequently, Frisch has modified her hypothesis to state that menarche in girls tends to occur at a critical constant body 